\title{
The Essence of Clinical Practice Guidelines for Ossification of Spinal Ligaments, 2019: 3. Diagnosis of OPLL
}

\author{
Hirotaka Chikuda
}

Department of Orthopaedic Surgery, Gumma University, School of Medicine, Gunma, Japan

\author{
Keywords: \\ OPLL, Diagnosis, Clinical characteristics
}

This article is the third part of the seven-article series, The Essence of Clinical Practice Guidelines for Ossification of Spinal Ligaments, published in the Spine Surgery and Related Research, Special Issue: Volume 5, Issue 5.

\section{Main Signs, Symptoms, and Neurological Characteristics of OPLL}

\section{Summary}

- OPLL can develop in the cervical spine, thoracic spine, or lumbar spine.

- There are considerable differences in the site, type, and degree of ligament ossification. In addition, the clinical symptoms caused by ligament ossification and their level of severity vary.

- As objective findings, accentuated tendon reflexes are often found in cervical and thoracic OPLL, whereas in lumbar OPLL, the tendon reflexes of the lower limbs are often reduced or disappear.

\section{Commentary}

At the time of the initial diagnosis of OPLL, patients present with radiculopathy, myelopathy, stiffness, and neck pain, while some patients have no subjective symptoms. Patients with OPLL may have serious paralysis caused by spinal cord compression. A study reported that all patients in whom ligament ossification caused a spinal canal stenosis rate $>60 \%$ exhibited myelopathy ${ }^{1)}$.

\section{Initial symptoms}

The mean age at symptom onset is about 50 years of $\operatorname{age}^{2}$. In cervical OPLL, initial symptoms often include localized neck symptoms such as neck pain and shoulder stiffness as well as numbness of the hands and feet, fine motor impairment of the fingers, and gait disturbance ${ }^{3)}$. In thoracic OPLL, initial symptoms include feeling of cold and numbness in the lower limbs, while in some patients, possible initial symptoms include tightness and a feeling of cold in the torso ${ }^{4)}$.

\section{Primary symptoms}

In cervical OPLL, symptoms include nuchal and cervical pain, pain and numbness in the upper limbs, pain and numbness in the lower limbs, motor impairment in the upper limbs, motor impairment in the lower limbs, and bladder and rectal disturbance. On the other hand, in thoracic OPLL, symptoms include numbness in the lower limbs and torso, gait disturbance, and bladder and rectal disturbance. Patients with thoracic OPLL are likely to have serious gait disturbance, with approximately one-third of patients unable to walk. Bladder and rectal disturbance in thoracic OPLL also tend to be more severe compared to that in cervical OPLL. These symptoms have a major impact on daily life.

\section{Objective findings}

In cervical OPLL, abnormal tendon reflex (59\%), sensory disturbance (56\%), and positive Hoffmann's reflex (41\%) were observed in the upper limbs, while abnormal tendon reflex $(57 \%)$, sensory disturbance $(37 \%)$, and positive Babinski sign (25\%) were observed in the lower limbs ${ }^{4}$. In thoracic OPLL, findings of sensory impairment in the lower limbs, deep tendon hyperreflexia, positive Babinski sign, and spastic paralysis are observed. Regarding urinary distur-

Corresponding author: Hirotaka Chikuda, chikuda-tky@umin.ac.jp

Received: June 10, 2021, Accepted: July 8, 2021

Copyright (C) 2021 The Japanese Society for Spine Surgery and Related Research 
bance, approximately $80 \%$ of patients with cervical OPLL present various urinary symptoms ${ }^{5}$.

\section{Characteristics of Imaging Findings of OPLL (Plain X-ray, MRI, and CT)}

\section{Summary}

- CT is useful when it is difficult to diagnose ossified lesions using plain X-ray.

- The degree of spinal cord compression on imaging and intramedullary signal intensity on MRI correlate with the severity of myelopathy.

- Double-layer signs on CT are useful for diagnosing dural ossification associated with postoperative spinal fluid leakage.

- The cross-sectional area of the spinal cord is decreased by flexion and extension of the cervical spine, with marked changes in the cross-sectional area of the spinal cord during flexion-extension found to correlate with severity.

\section{Commentary}

\section{Diagnosis of ossified lesions}

Cervical OPLL can be diagnosed using plain X-ray, which can be used to classify ossified lesions into four types: continuous type, segmental type, mixed type, and localized type ${ }^{2}$. CT and reconstructed CT images are useful for diagnosing ossified lesions. The reliability of cervical OPLL classification and diagnosis was found to be improved by concurrent use of CT. Thus, for the diagnosis of OPLL, it is recommended to perform plain X-ray in combination with $\mathrm{CT}^{8)}$. 3D-CT is also reported to be useful for evaluating the morphology of the ossified lesion and spinal cord compression caused by OPLL ${ }^{9)}$.

Many reports have indicated a relationship between the degree of spinal cord compression and myelopathy. In a study that examined patients who had undergone laminoplasty for cervical OPLL, the cross-sectional area of the spinal cord at the most stenotic level showed weak correlation with Japanese Orthopaedic Association (JOA) scores, although no correlation with Hirabayashi improvement rate was observed ${ }^{10)}$. Similarly, a study using 3D-CT revealed that the ossification occupancy rate in the spinal canal was an independent risk factor of the severity of myelopathy in patients with symptomatic cervical OPLL ${ }^{11)}$. A novel classification of OPLL based on CT findings was also proposed ${ }^{12)}$. In studies using MRI, the association between degree of spinal cord compression and clinical severity was reported ${ }^{13,14}$. A study examining the ossification occupancy rate found that all patients with an ossification occupancy rate $>60 \%$ exhibited myelopathy, while about half of the patients with an ossification occupancy rate of $<60 \%$ had myelopathy ${ }^{15}$.

\section{Dural ossification}

Dural ossification is occasionally observed in patients with OPLL. Recognition of dural ossification is important as it is a risk factor for surgery-related spinal fluid leakage. The double-layer sign on transverse CT is reportedly useful for diagnosing dural ossification ${ }^{16,17}$. With this sign, highdensity areas can be identified in the area of OPLL and dural ossification, with a low-density area present between these areas. The sensitivity and specificity of the doublelayer sign in the diagnosis of dural ossification were $55 \%$ and $96.9 \%$, respectively ${ }^{16}$.

\section{Changes in intramedullary signal intensity on MRI}

Intramedullary high intensity on T2 MRI was commonly found in patients with cervical OPLL. The level of hyperintensity signal appearance corresponded with the site of compression caused by ossification in all patients with continuous-type OPLL. It also corresponded to the intervertebral level in all patients with segmental-type OPLL ${ }^{18}$. Several studies reported an association between intramedullary changes and severity of myelopathy ${ }^{19-21}$. In a study using contrast-enhanced MRI, intramedullary enhanced area was observed in $4 \%$ of patients with cervical OPLL, but no relationship with preoperative symptoms was observed ${ }^{22}$.

\section{Dynamic factors and spinal compression}

Dynamic factors are involved in the condition of patients with myelopathy caused by cervical OPLL as the crosssectional area of the spinal cord is decreased with cervical spine in flexion and extension. Studies have shown that spinal cord is usually compressed more on extension than on flexion. However, the spinal cord is compressed more on flexion than on extension in patients with large occupancy rate (i.e., $>60 \%$ ) or kyphosis ${ }^{23,24)}$.

Conflicts of Interest: The author declares that there are no relevant conflicts of interest.

This is a part of The Essence of Clinical Practice Guidelines for Ossification of Spinal Ligaments (2019), which is listed below.

1. Epidemiology of OPLL, written by Tomohiko Hasegawa, MD, PhD, Hamamatsu University, School of Medicine, Japan. https://doi.org/10.22603/ssrr.20210096

2. Pathology of OPLL, written by Takashi Kaito, MD, $\mathrm{PhD}$, Osaka University Graduate School of Medicine, Japan. https://doi.org/10.22603/ssrr.2021-0074

3. Diagnosis of OPLL, written by Hirotaka Chikuda, MD, PhD, Gumma University, School of Medicine, Japan. https://doi.org/10.22603/ssrr.2021-0118

4. Treatment of Cervical OPLL, written by Toshitaka Yoshii, MD, PhD, Tokyo Medical and Dental University Hospital, Japan. https://doi.org/10.22603/ssrr.20210100 
5. Treatment of Thoracic OPLL, written by Shiro Imagama, MD, PhD, Nagoya University Graduate School of Medicine, Japan. https://doi.org/10.22603/ssrr.20210095

6. Diagnosis of OLF, written by Masao Koda, MD, PhD, University of Tsukuba, Japan. https://doi.org/10.22603/ ssrr.2021-0116

7. Treatment of Thoracic OLF, written by Kanji Mori, $\mathrm{MD}, \mathrm{PhD}$, Shiga University of Medical Science, Japan. https://doi.org/10.22603/ssrr.2021-0094

The original version of this clinical practice guidelines appeared in Japanese as Sekichu Jintai Kokkashou Shinryo Guidelines 2019, published by the Japanese Orthopaedic Association and the Japanese Society for Spine Surgery and Related Research, and its translated version in English appeared in the Journal of Orthopaedic Science 26 (2021) 145.

\section{References}

1. Matsunaga S, et al. The symptoms and diagnosis of cervical myelopathy caused by ossification of posterior longitudinal ligament. Jpn J Clin Exp Med. 1999;76(4):695-700. Japanese.

2. Tsuyama N. Ossification of the posterior longitudinal ligament of the spine. Clin Orthop. 1984;184:71-84.

3. Nishiura I, et al. A clinical examination of 182 patients with cervical ossification of the posterior longitudinal ligament. Spine Spinal Cord. 1994;7(12):1021-8.

4. Kawai S, et al. Clinical findings of ossification of the posterior longitudinal ligament. J Musculoskeletal Syst. 1990;3(6):567-72.

5. Hattori T, Yasuda K, Sakakibara R, et al. Micturitional disturbance in ossification of the posterior longitudinal ligament in the cervical spine. J Spinal Disord. 1990;3(4):285-7.

6. Kudo H, Yokoyama $T$, Tsushima E, et al. Interobserver and intraobserver reliability of the classification and diagnosis for ossification of the posterior longitudinal ligament of the cervical spine. Eur Spine J. 2013;22(1):205-10

7. Kawaguchi Y, Urushisaki A, Seki S, et al. Evaluation of ossification of the posterior longitudinal ligament by three-dimensional computed tomography and magnetic resonance imaging. Spine J. 2011;11(10):927-32.

8. Ito K, Yukawa Y, Machino M, et al. Spinal cord cross-sectional area during flexion and extension in the patients with cervical ossification of posterior longitudinal ligament. Eur Spine J. 2013;22 (11):2564-8

9. Lee N, Ji GY, Shin HC, et al. Usefulness of 3-dimensional measurement of ossification of the posterior longitudinal ligament
(OPLL) in patients with OPLL-induced myelopathy. Spine. 2015; 40(19):1479-86.

10. Kawaguchi Y, Matsumoto M, Iwasaki M, et al. New classification system for ossification of the posterior longitudinal ligament using CT images. J Orthop Sci. 2014;19(4):530-6.

11. Yone K, et al. Diagnosis and treatment of ossification of the posterior longitudinal ligament. Image evaluation of the spinal cord in ossification of the posterior spinal ligament. Orthopaedics. 1991; (40):29-36. Japanese.

12. Sawamura $S$, et al. The significance of measuring the form of the spinal cord using MRI. Orthopaedics. 1994;7(1):73-8. Japanese.

13. Matsunaga S, Nakamura K, Seichi A, et al. Radiographic predictors for the development of myelopathy in patients with ossification of the posterior longitudinal ligament: a multicenter cohort study. Spine. 2008;33(24):2648-50.

14. Chen Y, Guo Y, Chen D, et al. Diagnosis and surgery of ossification of posterior longitudinal ligament associated with dural ossification in the cervical spine. Eur Spine J. 2009;18(10):1541-7.

15. Yang H, Yang L, Chen D, et al. Implications of different patterns of "double-layer sign" in cervical ossification of the posterior longitudinal ligament. Eur Spine J. 2015;24(8):1631-9.

16. Fujiyoshi F, et al. MRI findings of cervical ossification of the posterior longitudinal ligament. Cent Jpn J Orthop Surg Traumatol. 1993;36(3):663-4.

17. Choi BW, Hum TW. Significance of intramedullary high signal intensity on magnetic resonance imaging in patients with cervical ossification of the posterior longitudinal ligament. Clin Orthop Surg. 2015;7(4):465-9.

18. Qizhi S, Lili Y, Ce W, et al. Factors associated with intramedullary MRI abnormalities in patients with ossification of the posterior longitudinal ligament. J Spinal Disord Tech. 2015;28(5):E304-9.

19. Kameyama $T$, et al. A study of spinal cord lesions in cervical spondylosis and ossification of the posterior longitudinal ligament using MRI. Clin Neurol. 1991;31(11):1177-81.

20. Yanase M, et al. Enhanced MRI of cervical ossification of the posterior longitudinal ligament. Orthop Traumatol. 1992;41(1):358-61.

21. Yoshii $\mathrm{T}$, Yamada $\mathrm{T}$, Hirai $\mathrm{T}$, et al. Dynamic changes in spinal cord compression by cervical ossification of the posterior longitudinal ligament evaluated by kinematic computed tomography myelography. Spine. 2014;39(2):113-9.

22. Ito $\mathrm{K}$, Yukawa $\mathrm{Y}$, Ito $\mathrm{K}$, et al. Dynamic changes in the spinal cord cross-sectional area in patients with myelopathy due to cervical ossification of posterior longitudinal ligament. Spine J. 2015;15 (3):461-6.

Spine Surgery and Related Research is an Open Access journal distributed under the Creative Commons Attribution-NonCommercial-NoDerivatives 4.0 International License. To view the details of this license, please visit (https://creativeco mmons.org/licenses/by-nc-nd/4.0/) 